\title{
Observation on colony formation of Microcystis aeruginosa induced by filtered lake water under laboratory conditions
}

\author{
Z. Yang $1,2,3 *$, F.X. Kong ${ }^{1}$, H.S. Cao ${ }^{1}$, X.L. Shi ${ }^{1}$ \\ ${ }^{1}$ Nanjing Institute of Geography and Limnology, the Chinese Academy of Sciences, 73 East Beijing Road, Nanjing 210008, China. \\ 2 Jiangsu Key Laboratory for Bioresource Technology, School of Biological Sciences, Nanjing Normal University, 122 Ninghai Road, Nanjing \\ 210097, China \\ ${ }^{3}$ Graduate School of the Chinese Academy of Sciences, Beijing 100039, China
}

\begin{abstract}
Microcystis aeruginosa mainly occurs as single cells in laboratory cultures, but in the colonial morph under natural conditions. This phenomenon suggests that some factors may be responsible for the typical occurrence of colonies in lakes. To demonstrate this possible effect, lake water with abundant or few zooplankton was filtered through a $0.15 \mu \mathrm{m}$ membrane filter, and used as test water. Microcystis aeruginosa was cultured in BG-11 medium, with or without test water. The results showed that filtered lake water with abundant zooplankton could induce colony formation in $M$. aeruginosa, whereas $M$. aeruginosa populations in the control and the treatment of filtered lake water with few zooplankton were still strongly dominated by unicells and paired cells, and no colony was formed. In the treatment of filtered lake water with abundant zooplankton, some colonies of several, dozens, and sometimes even hundreds of $M$. aeruginosa cells were formed. The unicells reduced to $53.4 \pm 6.5 \%$ and the proportion of cells in colonies increased from 0 to $24.5 \pm 4.6 \%$ of the populations. The mean number of cells per unit of $M$. aeruginosa in the treatment of filtered lake water with abundant zooplankton was promoted significantly compared with those of the control and the treatment of filtrated lake water with few zooplankton. In addition, no significant difference in growth rate was detected between the control and the treatments, regardless to the filtered lake water (i.e. with abundant zooplankton or with few zooplankton). Thus, we concluded that colony formation in our experiment was probably not associated with the nutrition difference but with some dissolved substances probably released from abundant zooplankton in the natural lake water, which may be one of the environmental factors responsible for the colonial form in M. aeruginosa.
\end{abstract}

Keywords : colony formation, filtered lake water, Microcystis aeruginosa, zooplankton, Lake Taihu.

\section{Introduction}

Freshwater algal blooms have become an important water quality problem in many countries, and Microcystis aeruginosa Kütz is commonly dominating in freshwater blooms. It is already known that $M$. aeruginosa occurs as colonial morph under natural conditions (Reynolds et al. 1981). However, when these colonies are isolated from the field and incubated in the artificial medium in laboratory, the characteristics of

\footnotetext{
* Corresponding author : E-mail : zhouyang@niglas.ac.cn
}

colonies disappear, and $M$. aeruginosa mainly exists as single cells and few paired cells (Bolch \& Blackburn 1996). Colonial isolates may loose their typical colonial appearance after some generations in the laboratory, suggesting that the factors responsible for the typical colonial form are absent in the culture media and exist in natural lake waters. To test this assumption, and more specifically to test whether colony formation is associated with zooplankton abundance, we used two types of filtered lake waters as test waters (one contained abundant zooplankton and the other contained few zooplankton), and $M$. aeruginosa was cultured in BG-11 medium with or without these test waters. 


\section{Materials and Method}

\begin{abstract}
Algae
M. aeruginosa Kütz was obtained from the Institute of Hydrobiology, Chinese Academy of Sciences. The alga was cultured axenically in liquid BG-11 medium (Rippka et al. 1979) in $1.0 \mathrm{~L}$ Erlenmeyer flasks at $25^{\circ} \mathrm{C}$ under fluorescent light at an intensity of $40 \mu \mathrm{E} \mathrm{m}^{-2} \mathrm{~s}^{-1}$ with a light-dark period of 12:12 hours. The alga in its exponential growth phase was used in this experiment.
\end{abstract}

\section{Filtered lake water}

Two types of natural lake water were sampled from two different sites in Lake Taihu (119 $53^{\prime} 45^{\prime \prime}$ $\left.120^{\circ} 36^{\prime} 15^{\prime \prime} \mathrm{E}, 30^{\circ} 55^{\prime} 42^{\prime \prime}-31^{\circ} 3350^{\prime \prime} \mathrm{N}\right)$ at the same time. Because of the role of wind power and hydrological factors, the distribution of zooplankton is not uniform during the sampling process. Among the two sampling sites, one showed abundant zooplankton (copepods $\approx 80$ ind $\cdot \mathrm{L}^{-1}$, cladocerans $\approx 400 \mathrm{ind} \cdot \mathrm{L}^{-1}$, rotifers $\approx 500$ ind $\cdot \mathrm{L}^{-1}$, protozoans $\approx 1000 \mathrm{ind} \cdot \mathrm{L}^{-1}$ ) and the other showed few zooplankton (copepods $<1$ ind $\cdot \mathrm{L}^{-1}$, cladocerans $<1$ ind $\cdot \mathrm{L}^{-1}$, rotifers $<3$ ind $\cdot \mathrm{L}^{-1}$, protozoans $<10$ ind $\cdot \mathrm{L}^{-1}$ ). The two different lake waters were filtered through $0.15 \mu \mathrm{m}$ membrane filters separately, and immediately used in the experiment. There was no significant difference in water quality between the two filtered lake waters.

\section{Experiment on colony induction}

A total of nine batches of $80 \mathrm{ml}$ of $M$. aeruginosa were transferred into cellulose plug stoppered $250 \mathrm{ml}$ Erlenmeyer flasks, followed by addition of $20 \mathrm{ml}$ of BG-11 medium (control), and $20 \mathrm{ml}$ of filtered lake water containing abundant zooplankton or $20 \mathrm{ml}$ of filtered natural lake water containing few zooplankton. The initial algal concentration (cell number) was approximately $8.5 \times 10^{6} \mathrm{cell}^{\mathrm{s}} \cdot \mathrm{ml}^{-1}$. The test was run in triplicate for 6 days at $25^{\circ} \mathrm{C}$ under fluorescent light at an intensity of $40 \mu \mathrm{E} \mathrm{m}^{-2} \mathrm{~s}^{-1}$ with a light-dark period of 12:12 hours. Samples were taken at regular intervals and the numbers of cells per unit were counted under a light-microscope by counting at least 600 units (i.e. unicells, two-celled as well as colonies). The mean number of cells per unit, the mean number of cells per colony (defined as more than two cells together), and the mean proportion of cells of unicells, two-celled, and colonies were computed from these counts, respectively. Growth rates of $M$. aeruginosa were estimated from increase in number of cells : $\mathrm{r}=\left(\ln N_{\mathrm{t}^{-}}\right.$ $\left.\ln N_{0}\right) \times \Delta \mathrm{t}^{-1}$, where $N_{\mathrm{t}}$ is the final algal number (cells $\bullet \mathrm{ml}^{-1}$ ), $N_{0}$ is the initial algal number and $\Delta \mathrm{t}$ is ex- perimental time (days). All data were presented as mean \pm 1 SD. Analysis of variance (ANOVA) was used to determine the significance of differences in the growth rate and mean number of cells per unit between control and the treatment.

\section{Results}

Colony formation of $M$. aeruginosa was observed in the treatment of filtered lake water with abundant zooplankton after 2 days. Some colonies of several, dozens, and sometimes even hundreds of $M$. aeruginosa cells were formed (Fig. 1A and B). However, M. aeruginosa populations in the control and the treatment of filtered lake water with few zooplankton were strongly dominated by unicells and paired cells and no colony was formed (Fig. 1C). The mean number of cells per

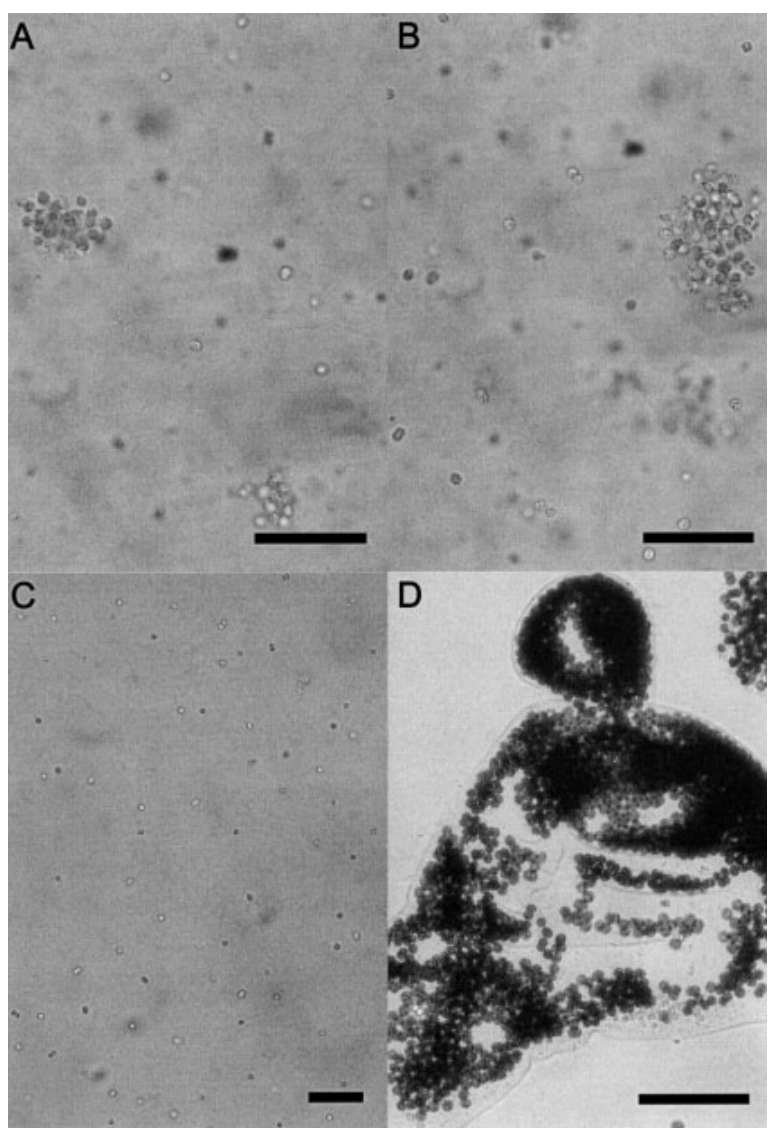

Fig. 1. Colonies of M. aeruginosa induced in the treatment of filtered lake water with abundant zooplankton (A and B), unicells of $M$. aeruginosa in the control (C), and large colonies collected from the field (D). Scale bars indicate $40 \mu \mathrm{m}$. 


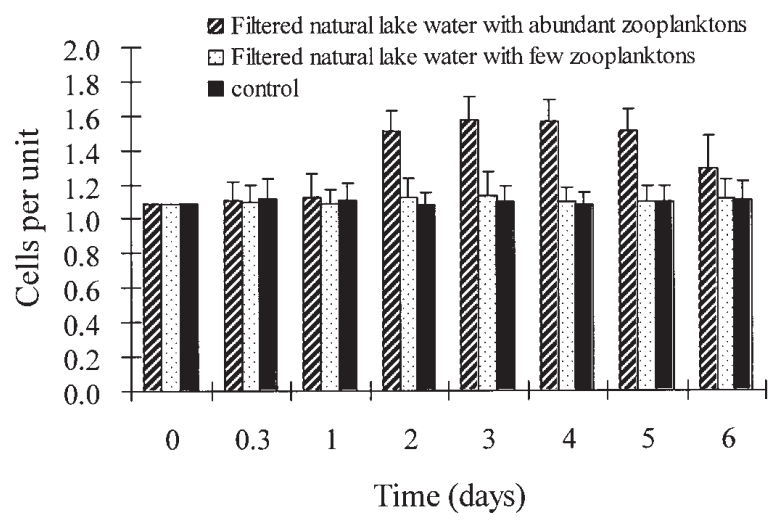

Fig. 2. Changes of the mean number of cells per unit of M. aeruginosa in different treatments. Vertical lines represent 1 SD.

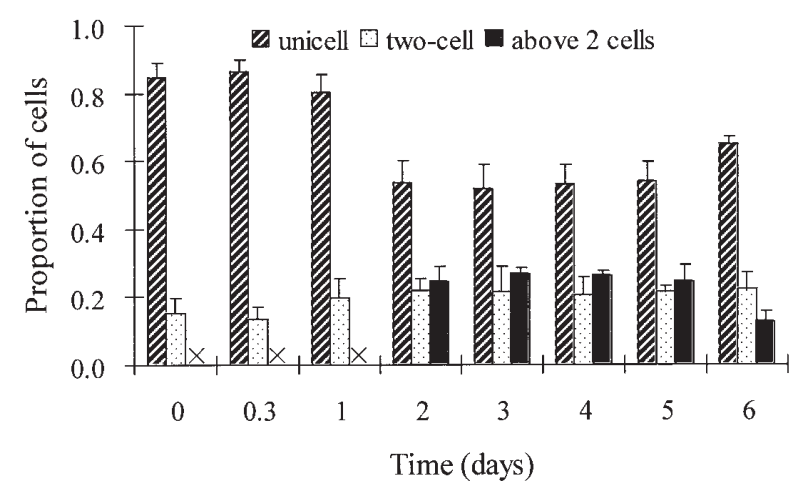

Fig. 4. Changes of the proportion of cells of different units in the treatment of filtered lake water with abundant zooplanktons. The symbol " $x$ " represents no data was detected. Vertical lines represent $1 \mathrm{SD}$.

unit of $M$. aeruginosa differed significantly between the control and the treatment of filtered lake water with abundant zooplankton $(\mathrm{P}<0.05)$, whereas there was no significant difference was detected between the control and the treatment of filtered lake water with few zooplankton $(\mathrm{P}>0.05)$ (Fig. 2). To express the colony formation in the treatment of filtered lake water with abundant zooplankton, the mean number of cells per colony was used, and its value was about 16 cells per colony (Fig. 3). At the beginning of the experiment, $M$. aeruginosa cultures were comprised of $84.7 \pm 4.3 \%$ unicells. After two days, the control populations remained dominated by unicells, while in the treatment of filtered lake water with abundant zooplankton the unicells reduced to $53.4 \pm 6.5 \%$ and the proportion of cells in colonies increased from 0 to

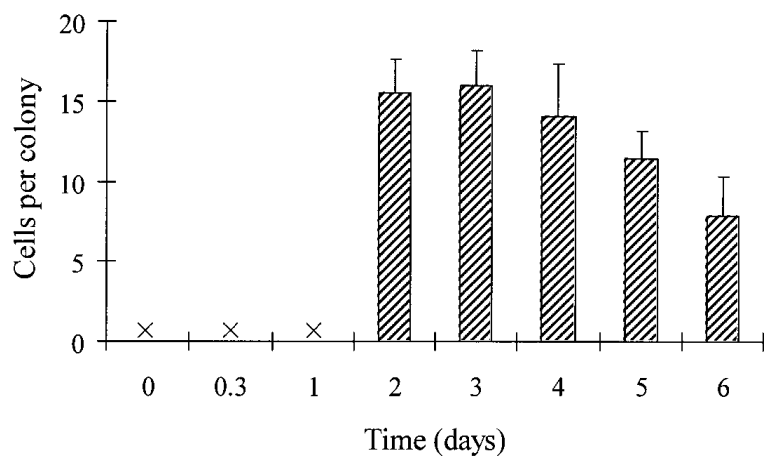

Fig. 3. Changes of the mean number of cells per colony of M. aeruginosa in the treatment of filtered lake water with abundant zooplanktons. The symbol " $x$ " represent no data was detected. Vertical lines represent $1 \mathrm{SD}$.

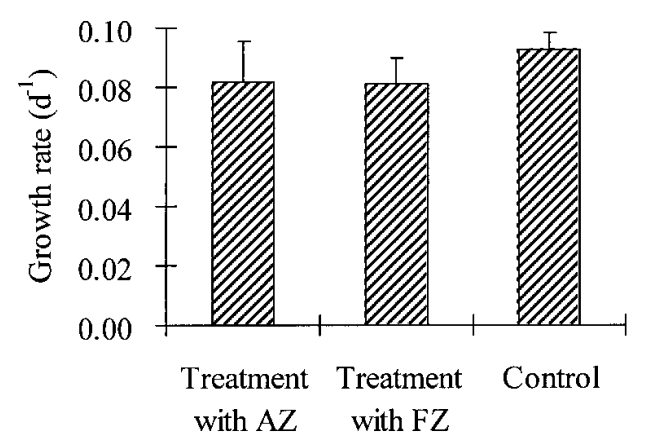

Fig. 5. Growth rate of M. aeruginosa in the treatment and in the control. Vertical lines represent 1 SD. (Treatment with AZ : Treatment of filtered lake water with abundant zooplankton ; Treatment with FZ : Treatment of filtered lake water with few zooplankton)

$24.5 \pm 4.6 \%$ of the population (Fig. 4). In addition, the proportion of paired cells showed no significant change throughout the experiments in the control and the two treatments. Although growth rate in the control was slightly higher than that in the two treatments, no significant difference was detected $(\mathrm{P}>0.05)$, regardless to the filtered lake water type (Fig. 5).

\section{Discussion}

The fact that colony formation was observed in $M$. aeruginosa in the treatment of filtered lake water with abundant zooplankton, whereas no colony was detected in the control and the treatment of filtered lake water with few zooplankton indicated that colony formation in M. aeruginosa was induced indirectly by dis- 
solved substances in the natural lake water. Furthermore, such colony formation is probably not associated with nutrition differences because there was no significant difference in growth rate between the two treatments and the control. Therefore, dissolved substances probably originating from abundant zooplankton may be one of the factors responsible for the colonial form. In the treatment of filtered lake water with abundant zooplankton, some colonies of dozens and hundreds of $M$. aeruginosa cells were formed. Two different mechanisms are involved in the colony formation; one mechanism is that colonies are formed when daughter cells of a recently divided cell remain in a regular arrangement during the reproductive process, and the other is that formation of colonies are adhesion of already existing single cells (Lürling 2003). The fact that the colonies formed in our experiment were arbitrary arrangements of cells suggested that colony formation in Microcystis was probably due to adhesion of already existing single cells. However, large colonies as those occurring in eutrophic lakes were not formed. The induced colonies, under artificial conditions in the laboratory, differed in number and shape from the colonies collected from the field (Fig. 1D). In the present experiment, the fact that about half of the populations still remained as unicells in the treatment of filtered lake water with abundant zooplankton indicated that the colony-inducing effect was very weak. The weaker colony-inducing effect may be due to axenic culture of $M$. aeruginosa used in the experiment. It is well known that the mucilage keeps the typical shape of a Microcystis colony and colonies of Microcystis in eutrophic lakes are commonly found with numerous bacteria embedded in the mucilage (Brunberg 1999, Pearl 1988, Boström et al. 1989). Therefore, it is likely that the colony formation of this species in natural water, associated with other factors such as bacteria, may give an advantage to $M$. aeruginosa to maintain colony mucilage (Brunberg 1999).

Several articles have already been published on colony formation of $M$. aeruginosa under laboratory conditions. Jang et al. (2003) and Ha et al. (2004) reported that colony formation in $M$. aeruginosa can be induced by zooplankton culture media filtrate ; Burkert et al. (2001) discovered colony formation in $M$. aeruginosa occasionally when they used dialyse tubing to investigate the interactions between $M$. aeruginosa and a potential grazer (Ochromonas sp.) ; Canini et al. (2001) reported that cultures of $M$. aeruginosa can be induced to form surface bloom. However, Fulton III \& Paerl (1987) reported that chemicals released from Daphnia ambigua appeared ineffective as colo- ny-inducing agents in Microcystis. Also, chemicals released from Daphnia magna were ineffective in inducing Microcystis colonies (Hesson \& Van Donk 1993). The above conflicting conclusions may be due to different experimental conditions, or to other unknown factors. Further clarification is thus needed. From our experiment, we can only conclude that some dissolved substances in the natural lake water, which were probably released from mixed zooplankton, may be one of the environmental factors responsible for the colonial form in $M$. aeruginosa. However, it is likely that the colony-inducing effect of different zooplankton may be not identical. Further studies are therefore needed to identify which zooplankton taxa (copepods, cladocerans, rotifers, protozoans) may play the most important role in inducing colony formation in $M$. aeruginosa.

\footnotetext{
Acknowledgements

We would like to thank Y. X. Wang and X. P. Chen for their assistance in the experiment. This study was supported by the National Natural Science Foundation of China (30400062), the Natural Science Foundation of Jiangsu Province (BK2004165), the State Key Fundamental Research and Development Program (No. 2002CB412305), Innovation Program of the Chinese Academy of Sciences (KZCX2-311).
}

\section{References}

Bolch C.J.S. \& Blackburn S. I. 1996. - Isolation and purification of Australian isolates of the toxic cyanobacterium Microcystis aeruginosa Kütz. J. Appl. Phycol., 8, 5-13.

Boström B., Pettersson A. K. \& Ahlgren I. 1989. - Seasonal dynamics of a cyanobacteria-dominated microbial community in surface sediments of a shallow eutrophic lake. Aquat. Sci., 51, 153178

Brunberg A.K. 1999. - Contribution of bacteria in the mucilage of Microcystis spp. (Cyanobacteria) to benthic and pelagic bacterial production in a hypereutrophic lake. FEMS Microbiol. Ecol., 29, 13-22.

Burkert U., Hyenstrand P., Drakare S. \& Blomqvist P. 2001. - Effects of the mixotrophic flagellate Ochromonas sp. on colony formation in Microcystis aeruginosa. Aquat. Ecol., 35, 9-17.

Canini A, Leonardi D \& Caiola M G. 2001. - Superoxide dismutase activity in the cyanobacterium Microcystis aeruginosa after surface bloom formation. New Phytol., 152, 107-116.

Fulton III R. S. \& Paerl H. W. 1987. - Effects of colonial morphology on zooplankton utilization of algal resources during blue-green algal (Microcystis aeruginosa) blooms. Limnol. Oceanogr., 32 : 634-644.

Ha K., Jang M. H. \& Takamura N. 2004. - Colony formation in planktonic algae induced by zooplankton culture media filtrate. $J$. Freshwater Ecol., 19, 9-16.

Hessen D. O. \& Van Donk E. 1993. - Morphological-changes in Scenedesmus induced by substances released from Daphnia. Arch. Hydrobiol., 127, 129-140.

Jang M. H., Ha K., Joo G. J. \& Takamura N. 2003. - Toxin production of cyanobacteria is increased by exposure to zooplankton. Freshwater Biol., 48, 1540-1550.

Lürling M. 2003. - Phenotypic plasticity in the green algae Desmodesmus and Scenedesmus with special reference to the induction of defensive morphology. Ann. Limnol. - Int. J. Lim., 39, 85-101. 
Pearl H. W. 1988. - Growth and reproductive strategies of freshwater bluegreen algae (cyanobacteria). In : Growth and Reproductive Strategies of Freshwater Phytoplankton (Sandgren, C.D., Ed.), pp. 261-315. Cambridge University Press, Cambridge.

Reynolds C.S., Jaworski G., Cmiech H. \& Leedale G. 1981. - On the annual cycle of the blue-green alga Microcystis aeruginosa Kütz Philos. T. Roy. Soc. London B, 293, 419-477.

Rippka R., Deruelles J., Waterbury J., Herdman M. \& Stanier R. 1979. - Generic assignments, strain histories and properties of pure cultures of cyanobacteria. J. Gen. Microbiol., 111, 1-61. 